\title{
Global Soot Growth Model
}

\author{
G. W. MULHOLLAND \\ Center for Fire Research \\ National Bureau of Standards \\ Gaithersburg, Maryland 20899, USA
}

\begin{abstract}
Analytical results for soot concentration, average particle size, and of the size distribution are obtained for a free radical soot growth model which includes a constant nucleation source, growth, and coagulation. Results are obtained with and without coagulation included and for a size independent growth rate as well as growth rate proportional to the surface area. Neither this model nor a nucleation pulse model is able to account for all the results on soot formation for shock tube and pyrolysis experiments.
\end{abstract}

\section{INTRODUCTION}

Soot produced in fires is a major concern. The emission of radiation from soot plays a dominant role in fire spread. The reduced visibility caused by soot is a significant impediment to persons escaping from fires. The deposition of soot in the respiratory tract is a potential health hazard especially in the case of repeated exposures by firemen. On the other hand, a low concentration of soot is adequate to activate a smoke detector alarm and thus provide a warning of a fire.

While there has been a large amount of experimental work concerning soot formation under a wide variety of conditions, as evidenced by some 388 references in a recent review article by Haynes and Wagner [1], there has been relatively $11 t t l e$ theoretical effort to integrate the various processes leading to soot formation and growth into a model capable of predicting soot concentration and particle size. Jensen [2] has developed such a model for predicting soot generation from methane fuel in an exhaust jet. The approach taken here is similar in spirit to Jensen's method though with less structure in the individual processes. The advantage of our model is that it can be solved analytically, and this allows a more general understanding of the effect of the various growth processes on the final particle size.

The model Includes the formation of soot nuclei, surface growth, and coagulation. The nucleation process is modeled as a thermal pyrolysis leading to a free radical species. The growth stage is first treated in a manner analogous to chain polymerization. Growth proportional to the surface area is also considered. For the combined growth model, the soot concentration, the average particle size, and the width of the size distributions are determined.

The model assumes a homogeneous isothermal system and for that reason the most direct validation is with shock tube experiments and pyrolysis experiments rather than with flame data. The nodel results are compared with the available data. 
FREE RADICAL SOOT GROWTH MODEL

This model is very similar to what is called polymerization without termination in the polymer 11terature. The first step involves the thermal pyrolysis of the fuel to yield free radical species.

$\mathrm{F} \stackrel{\mathrm{k}_{1}}{\rightarrow} \mathrm{R}_{1}+\mathrm{R}_{\mathrm{a}}$

where $R_{1}$ indicates the incipient soot nuclei and $R_{a}$ indicates a gaseous radical such as $\mathrm{H}$ atoms. The rate constant $\mathrm{k}_{1}$ is taken to have an activation energy, $\mathrm{E}_{\mathrm{a}}$. Of course, the chemistry leading to the soot precursor in an actual system is not a simple elementary reaction so this is a global expression of the kinetics.

The free radical $R_{1}$ initiates the chaln polymerization.

$R_{1}+F \stackrel{k_{2}}{\rightarrow} R_{2}$

$R_{j}+F \stackrel{k_{2}}{\rightarrow} R_{j+1}$

The symbols, $F, R_{1}, R_{j}$, etc. represent species in the equations above; we use the same symbols to represent concentration in the kinetic equations developed below. The rate constant $k_{2}$ is taken to be a constant independent of particle size. It is assumed that the fuel adds directly to the radical species. The polymer species $R_{j}$ are considered to be soot particles. We denote by $N$ the total number concentration of soot.

$N=\sum_{j=1}^{\infty} R_{j}$

The rate of change of $N$ depends only on the pyrolysis reaction.

$\mathrm{dN} / \mathrm{dt}=\mathrm{k}_{1} \mathrm{~F}$

The rate of change of the fuel concentration is obtained by adding up a11 the loss terms,

$\mathrm{dF} / \mathrm{dt}=-\mathrm{k}_{1} \mathrm{~F}-\mathrm{k}_{2} \mathrm{FN}$

In the small time 1imit, we replace $F$ with $F_{O}$ in eqs. (2) and (3) and obtain

$\mathrm{N}=\mathrm{k}_{1} \mathrm{~F}_{\mathrm{o}} \mathrm{t}$

$F_{0}-F=k_{1} F_{0} t+1 / 2 k_{1} k_{2}\left(F_{0} t\right)^{2}$

The fuel depletion, $F_{0}-F$, is also proportional to the soot volume concentration for this model, because there are only two types of species-fuel and soot. Equations (2) and (3) have also been solved exactly and the results plotted in figure 1 for the following conditions: initial fuel concentration of $2.5 \times 10^{17} \mathrm{~cm}^{-3}, \mathrm{k}_{2}=10^{-11} \mathrm{~cm}^{3} / \mathrm{s}$, and $\mathrm{k}_{1}=10^{-3} \mathrm{~s}^{-1}$. The linear portion of the curves correspond to eqs. (4) and (5). These expressions remain valid until the fuel becomes depleted by $10-20 \%$. The time dependence of the average volume can be determined from eqs. (4) and (5).

$\bar{v}=v_{0}\left(F_{0}-F\right) / N=v_{0}+1 / 2 v_{0} k_{2} F_{0} t$

where $v_{o}$ is the volume of a fuel molecule. 


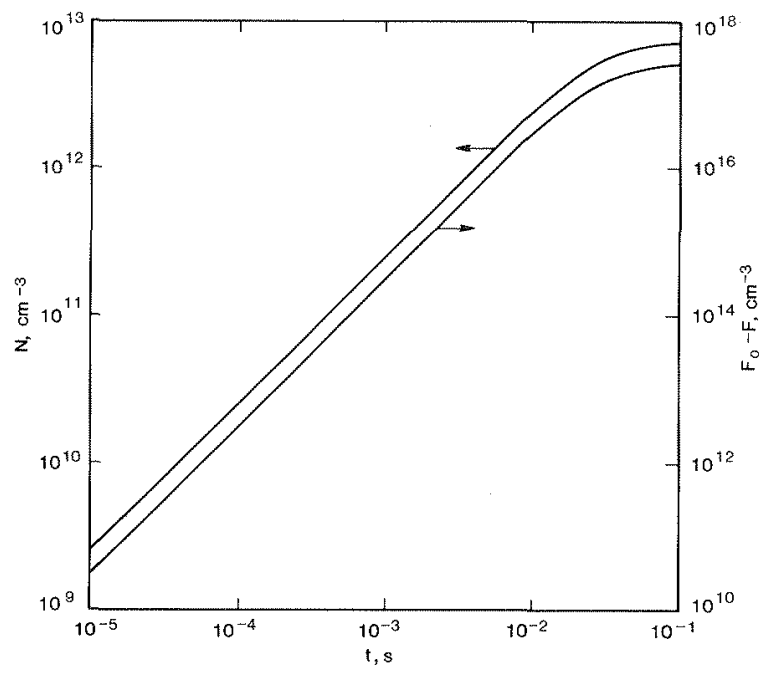

FIGURE 1. Number concentration, $N$, and fuel depletion, $F_{O}-F$, are plotted versus time for the free radical growth model.

For this model, the size distribution function, $R_{j}$, can also be obtained. The rate equations for the species $R_{j}$ are given by

$\mathrm{dR}_{1} / \mathrm{dZ}=\mathrm{k}_{1} / \mathrm{k}_{2}-\mathrm{R}_{1}$

$d R_{j} / d Z=R_{j-1}-R_{j}$

where $\mathrm{dZ}=\mathrm{k}_{2} \mathrm{Fdt}$. Solving this set of linear equations sequentially, we obtain

$\mathrm{R}_{j}=\frac{\mathrm{k}_{1}}{\mathrm{k}_{2}}\left[1-\sum_{i=0}^{j-1} \frac{\mathrm{z}^{i}}{i !} \mathrm{e}^{-\mathrm{z}}\right]$

As an example, the size distribution function is plotted in figure 2 for the same set of conditions as described above. The value of $j$ at which $R_{j}$ drops by a

factor of two, denoted by $j_{1}$, is given by $j_{1} \approx Z_{*}$. The value of $R_{j}$ changes rapidly about the value $j_{1}$ changing from $0.99 k_{1} / k_{2}$ to $0.01 k_{1} / k_{2}$ as $j$ increases from $j_{1}-2 \sqrt{j_{1}}$ to $j_{1}+2 \sqrt{j_{1}}$.

The average particle volume, $\bar{v}$, and $\sigma$, defined by $\left(\bar{v} 2-\bar{v}^{2}\right)^{1 / 2}$ for this size distribution are found to be,

$$
\begin{aligned}
& \bar{v}=v_{1} / 2 \\
& \sigma=\bar{v} / \sqrt{3}
\end{aligned}
$$

where $v_{l}$ corresponds to a particle with $j_{1}$ monomer units. The abrupt change in $R_{j}$ at $j=j_{l}$ is approximate as a step change in deriving eqs. (9) and (10). So, as is apparent from figure 2, the size distribution is broad with the width proportional to the mean volume. 


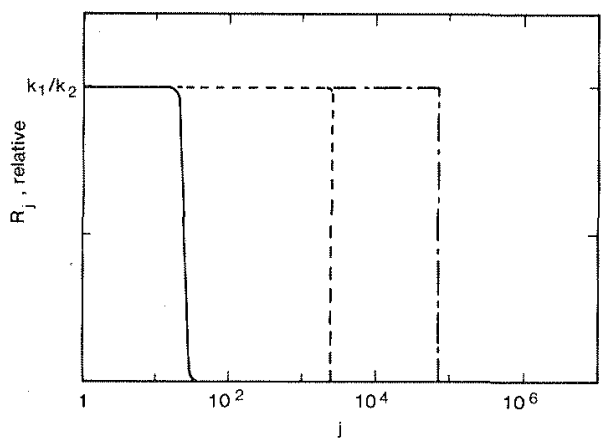

FIGURE 2. Size distribution function for the free radical growth model for $\mathrm{Z}=25\left(\mathrm{t}=10^{-5} \mathrm{~s}\right) \stackrel{\mathrm{C}}{\mathrm{C}}, \mathrm{Z}=2500\left(\mathrm{t}=10^{-3} \mathrm{~s}\right)-\cdots$, and $\mathrm{Z}=70,000$ $(t=0.1 \mathrm{~s}) \longrightarrow$

\section{GROWTH PLUS COAGULATION}

At the very high particle concentration typical of a flame environment, particles will collide and stick together in the coagulation process. The rate of decrease of the total number concentration, $\mathrm{N}$, is proportional to the square of the number concentration with coagulation coefficient designated by $\Gamma$.

$\mathrm{dN} /\left.\mathrm{dt}\right|_{\operatorname{coag}}=-\Gamma \mathrm{N}^{2}$

This represents a first order treatment of coagulation; a more detafled treatment would include the dependence of the coagulation coefficient on particle size such as the analysis by Dobbins and Mulholland [4]. Including coagulation, eq. (2) becomes

$\mathrm{dN} / \mathrm{dt}=\mathrm{k}_{1} \mathrm{~F}-\Gamma \mathrm{N}^{2}$

In the following analysis, we assume that the fuel is only slightly depleted so that $\mathrm{F}$ may be replaced by $\mathrm{F}_{\mathrm{o}}$ in eq. (11). Integrating eq. (11), we obtain

$N=N_{s s} \tanh (t / \tau)$

where $\mathrm{N}_{\mathrm{Ss}}=\left(\mathrm{k}_{1} \mathrm{~F}_{\mathrm{o}} / \mathrm{r}\right)^{1 / 2}$

$$
\tau=\left(k_{1} F_{0} \Gamma\right)^{-1 / 2}
$$

Substituting from eq. (12) into eq. (3) with $F$ replaced with $F_{o}$ on the right hand side and integrating, we obtain

$F_{0}-F=k_{I} F_{0} t+\frac{k_{2} F_{0}}{\Gamma} \log [\cosh (t / \tau)]$

The number concentration, $N$, and fuel depletion, $F_{0}-F$, for small time have the same form as the case of no coagulation. For long time, the number concentration approaches a steady state as the rate of formation is balanced by the loss rate from coagulation. It is convenient to use the following approximate form for the time dependence of $\mathrm{N}$ : 


$$
\begin{array}{ll}
N=N_{\text {ss }} t / \tau & t<\tau \\
N=N_{\text {sS }} & t>\tau
\end{array}
$$

Using this form for $\mathrm{N}$ in eq. (3) leads to the following expression for the fuel depletion:

$$
\begin{array}{ll}
F_{0}-F=k_{1} F_{0} t+1 / 2 k_{1} k_{2} F_{0}^{2} t^{2} & t<\tau \\
F_{0}-F=k_{1} F_{0} t+k_{2} N_{s s} F_{0}(t-\tau / 2) & t>\tau
\end{array}
$$

As seen in figure 3, the approximate expressions are valid in the limits of short and long time and, at worst, are about $15 \%$ greater than the correct value.

The rate equation for the species $R_{j}$ are given by

$$
\begin{aligned}
& d R_{I} / d t=k_{1} F_{0}-k_{2} R_{1}-2 \Gamma R_{1} N \\
& d R_{j} / d t=k_{2} F_{0} R_{j-1}-k_{2} F_{0} R_{j}+\Gamma \sum_{i+k=j} R_{i} R_{k}-2 \Gamma R_{j} N
\end{aligned}
$$

The kinetic equations for the first three moments of the distribution function, $N, V$, and $V_{2}$, are readily derived from eq. (18).

$$
\begin{aligned}
& \mathrm{dN} / \mathrm{dt}=\Sigma \mathrm{R}_{\mathrm{j}}=\mathrm{k}_{1} \mathrm{~F}_{\mathrm{o}}-\Gamma \mathrm{N}^{2} \\
& \mathrm{dV} / \mathrm{dt}=\mathrm{v}_{0} \sum_{j} \mathrm{R}_{j}=\mathrm{k}_{1} \mathrm{~F}_{0} \mathrm{v}_{0}+\mathrm{k}_{2} \mathrm{~F}_{0} \mathrm{~N} \mathrm{v}_{0} \\
& \mathrm{dV}_{2} / \mathrm{dt}=\mathrm{v}_{\mathrm{o}}^{2} \Sigma \mathrm{j}^{2} \mathrm{R}_{j}=\mathrm{k}_{1} \mathrm{~F}_{\mathrm{o}} \mathrm{v}_{\mathrm{o}}^{2}+2 \mathrm{I} \mathrm{v}^{2}+2 \mathrm{k}_{2} \mathrm{~F}_{\mathrm{o}} \mathrm{v}_{\mathrm{o}} \mathrm{V}
\end{aligned}
$$

The volume concentration $V$ is simply $v_{0}\left(F_{O}-F\right)$. In integrating eq. (19) to obtain $V_{2}$, we approximate $\mathrm{N}$ with $\mathrm{eq} .(16)$ and $V$ using the second term in eq. (17), which dominates the first for most cases of interest. For a similar reason, the first term in eq. (19) is dropped. The intensive quantities $\vec{v}$ and $\sigma$, derived from $\mathrm{N}, \mathrm{V}$, and $\mathrm{V}_{2}$, are glven by

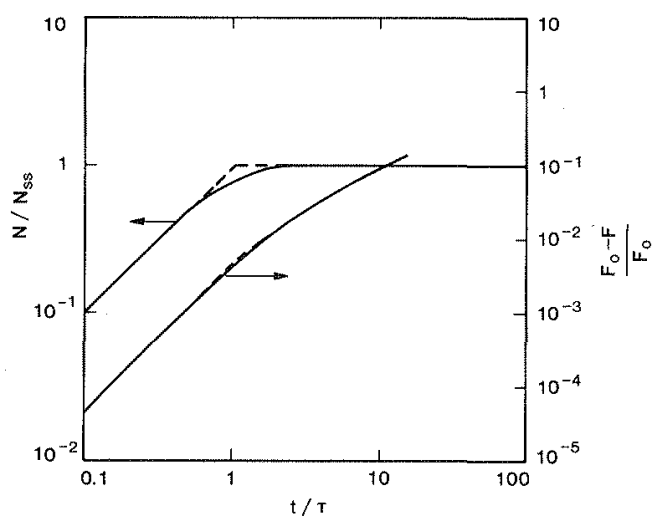

FIGURE 3. Reduced number concentration and reduced fuel depletion are plotted versus time for the free radical growth model with coagulation included. The dashed curves correspond to the approximations given by eqs. (16) and (17). 


$$
\begin{array}{ll}
\bar{v}=\frac{v_{0} k_{2} F_{0} \tau}{2}(t / \tau) & t<\tau \\
\bar{v}=v_{0} k_{2} F_{0} \tau(t / \tau-1 / 2) & t>\tau \\
\sigma=\frac{\left(v_{0} k_{2} F_{o} \tau\right)}{2 \sqrt{3}}(t / \tau)=\bar{v} / \sqrt{3} & t<\tau \\
\sigma=\left(v_{o} k_{2} F_{o} \tau\right) \sqrt{2 / 3}(t / \tau)^{3 / 2}=\sqrt{2 / 3}(t / \tau)^{1 / 2} \bar{v} & t>\tau
\end{array}
$$

The presence of coagulation causes the ratio $\sigma / \bar{v}$ to grow with time. Without coagulation, the ratio $\sigma / \bar{v}$ is constant. It can be seen from the study of Dobbins and Mulholland [4] that, with a constant nucleation source and no growth except by coagulation, one obtains $\sigma / \bar{v} \sim t^{1 / 2}$ for large time, just as in eq. (2l) above. Boisdron and Brock [5] have analyzed eq. (18) for the initial value problem where, at time $t$ equal zero, there are only species $R_{1}$ plus the growth species. The nucleation term, $\mathrm{k}_{1} F_{0}$, is not present in their analysis. Under this condition, the number concentration decreases with respect to time rather than increasing to a steady state value and $\sigma$ becomes equal to the average particle volume in the long time limit. We shall consider these results in relation to experimental results below.

\section{SURFACE GROWTH}

In the free radical growth mode1, it is assumed that there is only a single active site on each growing soot particle. There is experimental evidence for premixed flames [6] that the growth rate is proportional to the surface area. To consider this possibility, we take the growth rate for $R_{j}$ to be proportional to $j^{2 / 3}$. The model equations then become

$$
\begin{aligned}
& \mathrm{dR}_{j} / \mathrm{dt}=\mathrm{k}_{2}(j-1)^{2 / 3} \mathrm{R}_{j-1}-\mathrm{k}_{2}(j)^{2 / 3} \mathrm{R}_{j} \quad j>1 \\
& \mathrm{dR}_{1} / \mathrm{dt}=-\mathrm{k}_{1} \mathrm{~F}_{0}-\mathrm{k}_{2} \mathrm{R}_{1}
\end{aligned}
$$

The rate of change of the fuel concentration in the limt of small fuel depletion is given by

$\mathrm{dF} / \mathrm{dt}=-\mathrm{k}_{1} \mathrm{~F}_{\circ}-\mathrm{k}_{2} \mathrm{~F}_{\circ}\left[\sum_{j} \mathrm{R}_{j} j^{2 / 3}\right]$

The presence of the fractional exponent complicates the problem. We approximate the sum in eq. (23) as follows:

$$
\sum R_{j}\left(j^{2 / 3}\right)=N\left(\frac{F_{0}-F}{N}\right)^{2 / 3}
$$

The change in the growth rate expression does not affect the expression for $\mathrm{dN} / \mathrm{dt}$; eq. (2) is still valid. Substituting for the sum in eq. (23) and then taking the ratio of eq. (23) to eq. (2), we obtain

$\mathrm{dF} / \mathrm{dN}=-1-\mathrm{k}_{2} / \mathrm{k}_{1} \mathrm{~N}^{1 / 3}\left(\mathrm{~F}_{\mathrm{o}}-\mathrm{F}\right)^{2 / 3}$

We are primarily interested in the growth stage where the second term on the right hand side is large compared to the first. So dropping the first term and integrating, we find 
$F_{o}-F=\left(1 / 4 \frac{k_{2}}{k_{1}}\right)^{3} N^{4}$,
or
$F_{o}-F=(1 / 4)^{3}\left(k_{2} F_{0}\right)^{3} k_{1} F_{o} t^{4}$

The average volume is given by

$\bar{v}=v_{0}(1 / 4)^{3}\left(k_{2} F_{0} t\right)^{3}$.

We see that the particle growth is much more rapid than in the case of the free radical growth.

The steady state size distribution function obtained from eq. (22) is given by

$R_{j}=k_{1} / k_{2} F_{o} j^{-2 / 3}$

In the case of free radical growth treated in Section 2, the steady state concentration is independent of size. Using eq. (28) and assuming an upper size cutoff at $j=n$, we obtain the following expressions for $\bar{v}$ and $\sigma$ :

$\overline{\mathrm{v}}=\mathrm{v}_{\mathrm{o}} / 4 \mathrm{n}$

$\sigma=12 / \sqrt{112} \overline{\mathrm{v}}=1.13 \overline{\mathrm{v}}$

As in the free radical growth, $\sigma$ is proportional to $\vec{v}$ though, in this case, the coefficient is about twice as large as for free radical growth.

The effect of coagulation is considered by approximating the sum in eq. (23) using eq. (24) and then substituting the expression given by eq. (16) for N. Integrating, we obtain

$$
\begin{aligned}
F_{o}-F & =(1 / 4)^{3}\left(k_{2} F_{o} \tau\right)^{3} N_{s s}(t / \tau)^{4} & & t<\tau \\
& =(1 / 3)^{3}\left(k_{2} F_{o} \tau\right)^{3} N_{s s}(t / \tau-1 / 4)^{3} & & t>\tau
\end{aligned}
$$

The corresponding expressions for $\bar{v}$ are given by

$$
\begin{aligned}
\bar{v} & =v_{o}(1 / 4)^{3}\left(k_{2} F_{o} \tau\right)^{3}(t / \tau)^{3} & & t<\tau \\
& =v_{0}(1 / 3)^{3}\left(k_{2} F \tau\right)^{3}(t / \tau-1 / 4)^{3} & & t>\tau
\end{aligned}
$$

The fuel depletion is less rapid with coagulation included as a result of the smaller number of growth sites, but the average particle size is about two times larger. We are not able to solve for the $\sigma$ of the size distribution with the coagulation included.

\section{COMPARISON WITH EXPERIMENTS}

Flames are not homogeneous with respect to concentration and temperature; in addition, there are both growth and burnout processes taking place. The most direct experimental tests of the models are with shock tube and high temperature pyrolysis experiments.

In a shock tube study by Frenklach et al. [7], soot formation was studied as a function of fuel concentration, temperature, and time. The results for acetylene for $\mathrm{T}<2000 \mathrm{k}$ can be expressed in the form 
$F_{0}-F \sim e^{-E_{a} / R T} F_{o}^{a} t^{b}$.

From the data in series $D$ for times of $0.5,1.0,1.5$, and $2.0 \mathrm{~ms}$, we obtain a value of about 2.7 for b. From data at carbon atom concentrations of about $2.0 \times 1017 \mathrm{~cm}^{-3}$ and $5.0 \times 10^{17} \mathrm{~cm}^{-3}$, we obtain a value of 1.5 for a with a large uncertainty, \pm 0.5 , because of the limited range of data. Prado and Lahaye [8] measured the dependence of the soot volume fraction on the concentration of benzene in nitrogen at a temperature of $1383 \mathrm{k}$ for a 0.5 second residence time. Measurements were made at eight concentrations over the range 0.25 to $10 \%$ with a value of 1.5 for a. The free radical growth model without coagulation is the best compromise for the exponents $a$ and $b$ with values of 2.0 for both versus the experimental values of 1.5 and 2.7. The dependence of $F_{0}-F$ on $F_{0}$ and $t$ for each model is given in Table 1.

TABLE 1. Summary of Model Results ${ }^{a}$

Constant Nucleation Source ${ }^{b}$

\begin{tabular}{|c|c|c|c|}
\hline F.R.G. & $\begin{array}{l}\text { F.R.G. } \\
+ \text { Coag. } \\
\end{array}$ & S.G. & S.G. + Coag. \\
\hline$k_{1} F_{0} t$ & $\left(k_{1} F_{o}\right)^{1 / 2}$ & $k_{1} F_{0} t$ & $\left(k_{1} F_{0}\right)^{1 / 2}$ \\
\hline$k_{1} F_{0}^{2} t^{2}$ & $\mathrm{k}_{1}^{1 / 2} \mathrm{~F}_{\mathrm{o}}^{3 / 2}(\mathrm{t}-\tau / 2)$ & $k_{1} F_{0}^{4} t^{4}$ & $\mathrm{k}_{1}^{1 / 2} \mathrm{~F}_{0}^{7 / 2}(\mathrm{t}-\mathrm{\tau} / 4)^{3}$ \\
\hline$F_{0} t$ & $F{ }_{0}(t-\tau / 2)$ & $\mathrm{F}_{0}^{3} \mathrm{t}^{3}$ & $\mathrm{~F}_{0}^{3}(t-\tau / 4)^{3}$ \\
\hline
\end{tabular}

$\bar{v} / \sigma \quad \sqrt{3} \quad \sqrt{3 / 2}(\tau / t)^{1 / 2} \quad 1 / 1.13$

Nucleation Pulse

\begin{tabular}{|c|c|c|c|c|}
\hline $\mathrm{N}$ & $\mathrm{N}_{\mathrm{O}}$ & $t^{-1}$ & $\mathrm{~N}_{\mathrm{O}}$ & $t^{-1}$ \\
\hline$F_{0}-F$ & $\mathrm{~F}_{\mathrm{o}} \mathrm{t}$ & $F_{o} \log t$ & $\mathrm{~F}_{0}^{3 / 2} \mathrm{t}^{3}$ & $\mathrm{~F}_{\mathrm{o}}^{3 / 2} \mathrm{t}^{2}$ \\
\hline $\bar{v}$ & $\mathrm{~F}_{\mathrm{o}} \mathrm{t}$ & $F_{0} t \log t$ & $\mathrm{~F}_{\mathrm{o}}^{3 / 2} \mathrm{t}^{3}$ & $\mathrm{~F}_{\mathrm{o}}^{3 / 2} \mathrm{t}^{3}$ \\
\hline $\bar{v} / \sigma$ & $\sqrt{\mathrm{v}}$ & 1 & $-m$ & $-\infty$ \\
\hline
\end{tabular}

\footnotetext{
${ }^{a}$ Numerical factors are not included for $N$ and $F_{0}-F$, and the temperature Independent constant $k_{2}$ is not included.

bAbbreviations: F.R.G. - free radical growth; S.G. - surface growth; Coag. - coagulation.
} 
The pyrolysis experiments of Prado and Lahaye indicate that the number concentration is independent of residence time. This is consistent with the steady state result obtained in the model as a result of a balance between nucleation and coagulation. Prado and Lahaye interpret their results differently by stating that the constancy of the number concentration is a result of both nucleation and coagulation being completed by 0.1 seconds when their first sample is taken. For the conditions of pyrolysis experiment with a temperature of about $1400 \mathrm{~K}$ and a particle size of $50 \mathrm{~nm}$, we calculate a free molecular coagulation coefficient [9] of $3.4 \times 10^{-9} \mathrm{~cm}^{3} / \mathrm{s}$. For an initial number concentration of $10^{10} \mathrm{~cm}^{-3}$, the concentration would decrease by a factor of 70 over the two second residence time in the pyrolysis tube as a result of coagulation.

Prado and Lahaye found that while the average particle diameter changed by a factor of five as the fuel concentration was increased 40 fold, the ratio $\vec{D} / \sigma_{D}$ had a constant value of about 5.5. To compare with our results in Table 1 , we compute $\bar{v} / \sigma_{y}$ based on the assumption that the size distribution is Gaussian with respect to particle diameter. We obtain

$\frac{\bar{v}}{\sigma_{v}}=\frac{k\left(1+3 / k^{2}\right)}{\left(9+36 k^{-2}+15 k^{-4}\right)^{1 / 2}}$

where $k=\bar{D} / \sigma_{D}$. For a value of 5.5 for $k$, we obtain $D / \sigma_{V}$ equa1 1.89. Again, the free radical growth model without coagulation gives the best agreement with this experimental result.

The growth species in the models presented is assumed to be the fuel molecule. If, instead, the growth species were acetylene, then for fuels other than acetylene, the pyrolysis reactions leading to the growth species must be included in the model.

The activation energy, $E_{a}$, determined by Frenklach et al. [7] for acetylene was about $126 \mathrm{~kJ} / \mathrm{mol}$. This is to be compared with a value of about $500 \mathrm{~kJ} / \mathrm{mol}$ for the free radical growth model. Including coagulation reduces the model prediction by a factor of two, but, as pointed out above, the other aspects of the model agree better with the free radical growth without coagulation.

\section{DISCUSSION}

The free radical growth model contains a chemical nucleation with an Arrhenius type kinetics. For constant temperature and small fuel depletion, the nucleation rate is constant. This differs from the short nucleation pulse predicted by homogeneous nucleation theory [10]; in this case, the nucleation is turned off by a slight decrease in the concentration of the condensing vapor. As a limiting case, the nucleation is assumed to be completed before the surface growth and coagulation begin. In Table 1 we have included the predictions for such a case which corresponds to a fixed initial number concentration. A similar model was described by Boisdron and Brock [5]. The best agreement between the mode1 and the shock tube and pyrolysis data for soot concentration is for the surface growth case. This is in contrast to the constant nulceation source where the best agreement is for free radical growth (rate constant $k_{2}$ independent of particle size). The narrower size distribution predicted for the nucleation pulse case appears to agree better with the available data [8], though the relatively constant number concentration observed by Prado and Lahaye [8] agrees better with the prediction of the constant nucleation source with coagulation included. At the high concentration typically observed, coagulation is surely important unless there is a very small sticking coefficient for colliding particles. 
So it is seen that no one of these models is consistent with all the experimental observations. Major improvements in our understanding of soot growth will require Innovative experimental approaches coupled with theoretical analyses. There is a need for data on the time dependence of the nucleation process. The nucleation rate and duration probably have the largest effect on the amount of soot produced and its size distribution. In order to better understand the growth processes, information is needed in regard to the sticking probability for gas-particle and particle-particle collisions as a function of particle size. The viscosity of the growing particles is required to model the transition from coalescence to agglomeration.

\section{REFERENCES}

[1] Haynes, B.S. and Wagner, H. Gg., Soot formation, Prog. Energy Combust. Sci, 7, $229(1981)$

[2] Jensen, D.E., Prediction of soot formation rates: a new approach, Proc. R. Soc. Lond. A. 338,375 (1974).

[3] Allen, P.E.M. and Patrick, C.R., Kinetics and Mechanisms of Polymerization Reactions, 162, Wiley, NY (1974).

[4] Dobbins, R.A. and Mulholland, G.W., Interpretation of optical measurements of flame generated particles, Combustion Science and Technology, 40, 175 (1984).

[5] Boisdron, Y. and Brock, J.R., Particle growth processes and initial particle size distributions, Assessment of Airborne Particles

(Mercer, T.T., Morrow, P.E. and Stöber, W.) 129, Thomas, Springfleld, IL (1972).

[6] Harris, S.J. and Weiner, A.M., Surface growth of soot particles in premixed ethylene/air flames, Combustion Science and Technology 31, 155 (1983).

[7] Frenklach, M., Taki, S., Durgaprasad, M.B. and Matula, R.A., Soot formation in shock-tube pyrolysis of acetylene, allene, and 1,3-butadiene, Combust. Flame 54, 81 (1983).

[8] Prado, G. and Lahaye, J., Physical aspects of nucleation and growth of soot particles, Particulate Carbon Formation During Combustion (edited Siegla, D.C. and Smith, G.W.) 143, Plenum Press, NY (1981).

[9] Friedlander, S.K., Smoke, Dust and Haze, 179, Wiley, NY (1977).

[10] Frenke1, J., Kinetic Theory of Liquids, Dover, NY (1955). 